Oncology

\title{
Transarterial Chemoembolization in Combination with a Molecular Targeted Agent: Lessons Learned from Negative Trials (Post-TACE, BRISK-TA, SPACE, ORIENTAL, and TACE-2)
}

\author{
Masatoshi Kudo Tadaaki Arizumi \\ Department of Gastroenterology and Hepatology, Kindai University Faculty of Medicine, Osaka-Sayama, Japan
}

\section{Keywords}

Hepatocellular carcinoma · Transarterial

chemoembolization - Clinical trial · Molecular targeted

agent · Sorafenib · Brivanib · Orantinib

\begin{abstract}
The multikinase inhibitor sorafenib is the first oral molecular targeted agent with proven prognostic benefit in unresectable advanced hepatocellular carcinoma (HCC). However, as with other drugs, sorafenib has its limitations, and various clinical trials have been conducted to develop novel molecular targeted agents for use alone or in combination with existing locoregional therapies. Despite this, clinical trials of molecular targeted agents combined with transarterial chemoembolization (TACE) have not reported major treatment outcomes to date. In this review, we describe previous clinical trials of combination therapy with TACE and a molecular targeted agent in patients with unresectable HCC.
\end{abstract}

(c) 2017 S. Karger AG, Basel

\section{Introduction}

The survival benefit of sorafenib in unresectable advanced hepatocellular carcinoma (HCC) was demonstrated in the Sorafenib Hepatocellular Carcinoma As-

\section{KARGER}

(C) 2017 S. Karger AG, Basel

E-Mail karger@karger.com

www.karger.com/ocl sessment Randomized Protocol (SHARP) and Asia-Pacific studies [1,2]. In 2007, sorafenib was approved in the EU and USA, where it became a first-line agent for unresectable advanced HCC. Transarterial chemoembolization (TACE) is the first-line treatment option for intermediate-stage HCC [3-6]; however, repeated TACE sessions tend to impair liver functional reserve. Reducing the frequency of TACE, which is generally repeated upon tumor progression, is a challenging issue in the treatment of patients with intermediate-stage HCC. To address this issue, previous trials combined molecular targeted agents with TACE; however, the safety and efficacy of this combination could not be demonstrated, and none of the combination therapies is currently recommended.

In the SHARP study, subanalysis showed that the hazard ratio (HR) for overall survival (OS) in patients with HCC with no vascular invasion or extrahepatic spread, which are regarded as good indications for TACE, was 0.52 and, thus, extremely good in the sorafenib group. The median survival of these patients was extended approximately 1.5 -fold [7], suggesting that the addition of sorafenib as adjuvant therapy to TACE improves prognosis. The combination of TACE with sorafenib does not simply represent the administration of 2 types of treatment; the combination is expected to extend the period during which TACE controls tumor progression because sorafenib strongly suppresses post-TACE tumor recur-

Prof. Masatoshi Kudo

Department of Gastroenterology and Hepatology Kindai University Faculty of Medicine

377-2 Ohno-Higashi, Osaka-Sayama, Osaka 589-8511 (Japan)

E-Mail m-kudo@med.kindai.ac.jp 
rence and progression by inhibiting hypoxia-induced angiogenesis. In addition, the detrimental effect on liver function may be suppressed by reducing the frequency of TACE. Sorafenib combined with TACE is expected to improve the survival of patients with intermediate-stage HCC, which is the goal of TACE, by extending the time to progression (TTP) to the advanced stage while maintaining liver function [8].

\section{The Post-TACE Study}

\section{Background}

TACE is used as a first-line treatment for unresectable HCC; however, the procedure is hardly curative in clinical practice and needs to be repeated multiple times. Repeated TACE may promote the deterioration of liver function. Based on the apparent role of angiogenesis and the upregulation of vascular endothelial growth factor (VEGF) associated with tumor recurrence after TACE, a study was designed with the objective of delaying the recurrence of HCC and prolonging OS by administering sorafenib after TACE. Sorafenib is an inhibitor of several tyrosine kinases involved in the growth of tumor cells and angiogenesis, such as Raf, VEGF receptor, and platelet-derived growth factor (PDGF) receptor. This placebo-controlled phase III study designated the Post-TACE study was conducted in Japan and Korea [9].

\section{Methods}

Patients with unresectable HCC and Child-Pugh A liver function who had imaging findings of objective treatment response (complete response or partial response) at 1-3 months after TACE were included. These patients were randomly assigned to 2 groups for sorafenib or placebo administration. The primary and secondary endpoints of the study were TTP and OS, respectively.

\section{Results}

A total of 458 patients ( 387 from Japan and 71 from Korea) were registered to the study between April 2006 and July 2009. These patients were randomized to the sorafenib $(n=229)$ or placebo $(n=229)$ arms. The median TTP, the primary endpoint of the study, was 5.4 and 3.7 months in the sorafenib and placebo groups, respectively (HR 0.87 [95\% CI 0.70-1.09]; $p=0.252$ ) (Table 1). The median OS, the secondary endpoint of the study, was 29.7 months in the sorafenib group, whereas a median value was not reached in the placebo group (HR 1.06 [95\% CI 0.69-1.64]; $p=0.790$ ). A subanalysis in Japanese patients showed that the median TTP was 3.9 and 3.7 months in the sorafenib and placebo arms, respectively, with a HR of 0.94 and no significant intergroup difference. In Korean patients, however, the HR was 0.38 and TTP was clearly longer in the sorafenib group.

\section{Possible Reasons for Failure}

In this study, the primary endpoint TTP did not differ significantly between the groups. One possible reason for this is the timing of sorafenib administration. TACE promotes the production of VEGF by triggering ischemic conditions. Therefore, for sorafenib to produce the expected effects, it may be necessary to inhibit angiogenesis occurring soon after TACE. However, because the study included only patients who responded to TACE, approximately $60 \%$ of the patients in the sorafenib group underwent drug treatment 9 weeks after TACE. This long waiting period might have contributed to the loss of the additive effect of sorafenib.

In addition, the mean daily dose in the sorafenib group was as low as $386 \mathrm{mg}$ because of many cases of dose reduction (73\%) and interruption (91\%). By contrast, the mean daily dose of sorafenib was $797 \mathrm{mg}$ in the SHARP study, and the occurrence of dose reduction (26\%) and interruption (44\%) was low. In the Asia-Pacific study, the mean daily dose of sorafenib was $795 \mathrm{mg}$, with a low occurrence of dose reduction (31\%) and interruption (43\%), and the study outcomes were markedly different from those of the Post-TACE study. The Post-TACE study was conducted before the results of the SHARP study were revealed to be positive, and Japanese patients did not participate in the SHARP or Asia-Pacific study; therefore, physicians in the Post-TACE study who may have been unfamiliar with the management of the side effects of sorafenib, especially hand-foot skin reaction, used the drug at low doses and short duration, resulting in poor treatment outcomes.

Although no significant intergroup difference was observed between Japan and Korea, significant differences in TTP were observed between Korea and Japan. This could be attributed to the considerably longer treatment duration in Korean patients than in Japanese patients (median 31 vs. 16 weeks), which generated a good HR. Furthermore, patient backgrounds were different between the 2 countries, with a higher proportion of elderly patients and a greater number of lesions in Japanese than in Korean patients. 
Table 1. Clinical trials of TACE combined with molecular targeted agents

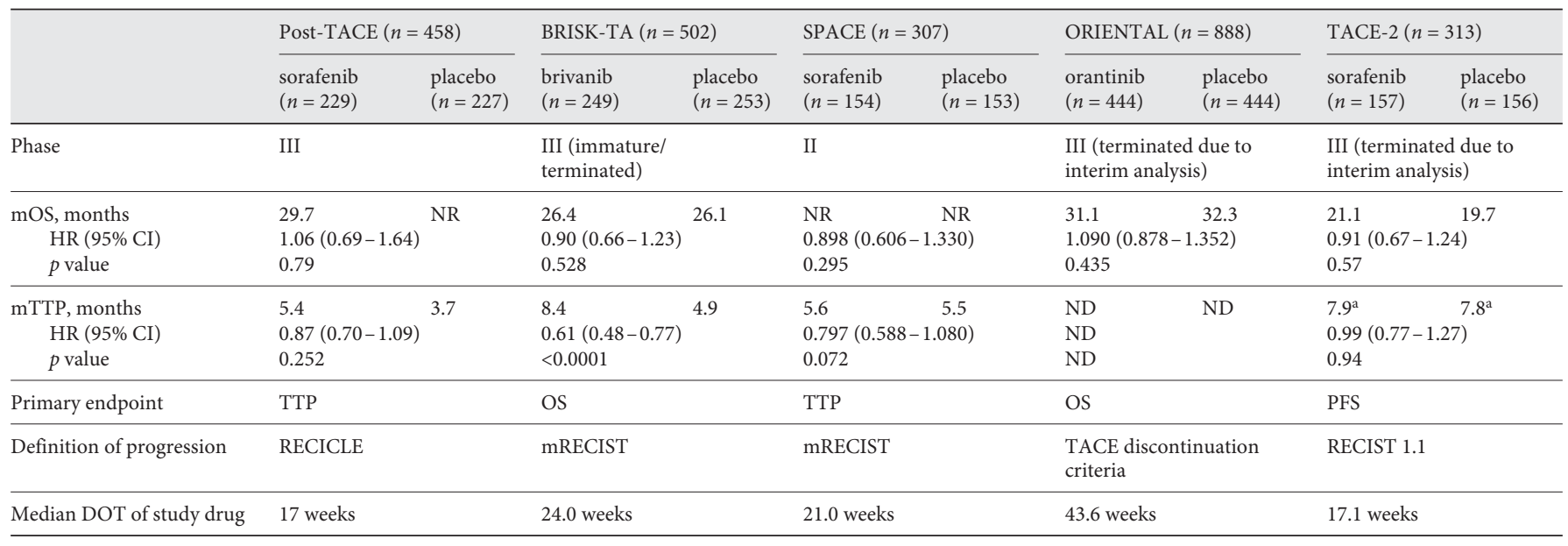

TACE, transarterial chemoembolization; mOS, median overall survival; NR, not reached; HR, hazard ratio; mTTP, median time to progression; ND, no data; PFS, progression-free survival; DOT, duration of treatment. ${ }^{\text {a }}$ Progression-free survival was used in the TACE-2 study.

\section{The BRISK-TA Study}

\section{Background}

Brivanib inhibits the signal transduction pathway of fibroblast growth factor receptor in addition to the VEGF receptor, and its mechanism of action is, therefore, different from that of sorafenib. Based on the action of brivanib, a phase III study was designed to investigate the efficacy and safety of the drug as an adjuvant therapy to TACE in patients with unresectable HCC [10].

\section{Methods}

At 83 institutes in 12 countries, a total of 502 patients were treated with TACE and randomly allocated to receive brivanib $(n=249)$ or placebo $(n=253)$. The period between TACE and drug administration ranged from 48 $\mathrm{h}$ to 21 days. TACE was repeated in patients receiving insufficient treatment and in those showing tumor progression or the development of a new lesion. Drug administration was stopped 2 days before the second or later TACE session and was resumed 3-21 days after TACE. The primary endpoint was OS, and secondary endpoints were time to disease progression, which is a composite endpoint, time to extrahepatic spread or vascular invasion (TTES/VI), and the number of TACE sessions. In the study, extrahepatic metastasis/spread, vascular invasion, death, exacerbation to Child-Pugh $\mathrm{C}$, and a reduction in performance status by 2 points were defined as disease progression.

TACE in Combination with a Molecular Targeted Agent

\section{Results}

Between August 2009 and August 2012, 502 patients were allocated to receive brivanib $(n=249)$ or placebo $(n=253)$. The median OS, the primary endpoint of the study, was 26.4 and 26.1 months in the brivanib and placebo arms, respectively (HR 0.90 [95\% CI 0.66-1.23]; $p=0.5280$ ) (Table 1 ). Regarding the secondary endpoints, the median time to disease progression was 12.0 and 10.9 months in the brivanib and placebo arms, respectively (HR 0.94 [95\% CI 0.72-1.22]; $p=0.6209$ ). However, the brivanib group had a longer TTES/VI (HR 0.64 [95\% CI 0.45-0.90]; $p=0.0096$ ) and fewer TACE sessions (HR 0.72 [95\% CI 0.61-0.86]; $p=0.0002$ ). The median TTP was 8.4 months in the brivanib group and 4.9 months in the placebo group; the former had a significantly longer TTP (HR 0.61 [95\% CI 0.48-0.77]; $p<$ $0.0001)$. The response rate per mRECIST [11] was 48 and $42 \%$ in the brivanib and placebo groups (odds ratio 1.28 [95\% CI $0.90-1.83]$ ), with 22 and $11 \%$ for complete response and 9 and $18 \%$ for progressive disease (PD), respectively.

\section{Possible Reasons for Failure}

The survival benefit of brivanib was not demonstrated in a phase III trial comparing OS noninferiority between brivanib and sorafenib as a first-line treatment for unresectable advanced HCC (the BRISK-FL study) and another phase III study that compared brivanib and placebo as a second-line treatment following sorafenib (the BRISK-PS study). Consequently, the BRISK-TA study

Oncology 2017;93(suppl 1):127-134 DOI: $10.1159 / 000481243$ 
was terminated 2 years earlier than originally planned, after enrolling only 502 patients, which was a markedly smaller cohort than the originally planned 870 patients. Although the primary endpoint OS did not differ significantly between the groups, TTES/VI, TTP, and response rate were significantly different, suggesting that brivanib delays tumor progression and metastasis. Because of the early termination of the study, in addition to patients, the number of deaths (needed to calculate the primary endpoint OS) was 164 , accounting for $32 \%$ of the initially anticipated number of deaths (520). This early termination also makes evaluation of drug efficacy difficult; however, the intergroup differences in TTES/VI and TTP, as well as the reduced number of TACE sessions, suggest that brivanib had a positive anticancer effect. If the study had been continued, it might have met its primary endpoint of prolonging overall survival.

\section{The SPACE Study}

\section{Background}

The SPACE study, which involved the use of drugeluting beads loaded with doxorubicin (DEBDOX), was conducted at 85 institutes in 13 countries, but not in Japan. This phase II study investigated the safety and efficacy of TACE with doxorubicin-eluting beads (DEBTACE) combined with sorafenib or placebo in patients with unresectable intermediate-stage (BCLC stage B) HCC. Based on the findings of the Post-TACE study indicating that VEGF production is upregulated in response to TACE-induced ischemia, which suggests that early administration of sorafenib is necessary, in the SPACE study, TACE was performed after the initiation of sorafenib treatment [12].

\section{Methods}

Patients registered at 85 institutes in 13 countries were randomly allocated to receive sorafenib or placebo. Sorafenib or placebo was administered 3-7 days before DEB-TACE. After the first DEB-TACE, the second and latter DEB-TACE procedures were performed at 3, 7, and 13 months and every 6 months thereafter. The primary endpoint was TTP, and the secondary endpoints were OS, TTES/VI, time to untreatable progression (TTUP) by TACE, and safety. The criteria for untreatable progression by TACE were defined as the occurrence of vascular invasion or extrahepatic spread, sustained ascites, ChildPugh class B, Eastern Cooperative Oncology Group (ECOG) performance status score of $\geq 2$, and platelet count of $<60,000 / \mu \mathrm{L}$. A 1 -tailed test was used to analyze the differences between the groups, with significance set at $15 \%(\alpha=0.15)$.

\section{Results}

Of 307 patients, 154 received sorafenib and 153 received placebo. The median TTP, the primary endpoint of the study, was 169 and 166 days in the sorafenib and placebo groups, respectively, thereby achieving the primary endpoint (HR 0.797 [95\% CI 0.588-1.08]; $p=0.072$ ) (Table 1). However, the secondary endpoints of OS and TTES/VI did not reach the median value in both groups (HR 0.898 [95\% CI 0.606-1.33]; $p=0.295$ for OS, and HR 0.621 [95\% CI $0.321-1.20$ ]; $p=0.076$ for TTES/VI). The median TTUP was 95 and 224 days in the sorafenib and placebo groups, respectively (HR 1.586 [95\% CI $1.200-$ 2.096]; $p=0.999$ ). The number of patients with TACEuntreatable progression was 110 and 96 in the sorafenib and placebo groups, respectively. The frequency of DEBTACE was once in $35.9 \%$ of sorafenib patients and $19.2 \%$ of placebo patients. Comparison between Asian and nonAsian patients showed that the proportion of Asian patients who underwent DEB-TACE once was $24.1 \%$ in the sorafenib group and $22.0 \%$ in the placebo group, and among non-Asian patients it was $42.4 \%$ in the sorafenib group and $17.8 \%$ in the placebo group. TTP in Asian patients was 24 months in the sorafenib group and 16.1 months in the placebo group, and TTP in non-Asian patients was 25.0 months in the sorafenib group and 24.0 months in the placebo group.

\section{Possible Reasons for Clinically Unmeaningful Results}

The SPACE study achieved the primary endpoint of TTP; however, no significant difference was observed in the secondary endpoint of OS. Similarly, TTUP did not show significant differences or was considerably shorter in the sorafenib group, inconsistent with the outcome initially anticipated. In this study, DEB-TACE was performed at 3, 7, and 13 months and every 6 months thereafter using the method-designated scheduled TACE, in which TACE is performed at fixed intervals. Even when intrahepatic lesions responded favorably to TACE, unnecessary repetition of TACE might have impaired liver function or increased the side effects of sorafenib. Unlike scheduled TACE, TACE is performed when needed in Japan using a method designated "on-demand TACE."

In this study, many patients had fewer TACE sessions, which may have occurred because the criteria for TACEuntreatable progression were not appropriate. Although
130

Oncology 2017;93(suppl 1):127-134 DOI: $10.1159 / 000481243$
Kudo/Arizumi 
vascular invasion and extrahepatic spread are adequate criteria, Child-Pugh B, sustained ascites, and a platelet count of $<60,000 / \mu \mathrm{L}$ may not indicate that patients are untreatable with TACE. The Japan/Korea Post-TACE study suggested that sorafenib might extend TTP if sorafenib is administered for a long period of time; however, in the SPACE study, sorafenib administration was terminated early based on the criteria for TACE-untreatable progression.

In addition, treatment duration of sorafenib was too short, i.e., only 21 weeks. In Asian patients, median treatment duration of sorafenib was 30 weeks, resulting in a favorable HR of $0.720(p=0.078)$, whereas in non-Asian patients, median treatment duration of sorafenib was just 17.4 weeks, resulting in a poor HR of $0.865(p=0.243)$. This implies that treatment duration is very important in the TACE combination trial similar to the Post-TACE study.

\section{ORIENTAL Study}

\section{Background}

A phase I/II study showed promising efficacy and safety of orantinib, a molecular targeted agent that inhibits VEGF, PDGF, and fibroblast growth factor receptors in patients with advanced HCC [13]. In light of the promising outcomes, a phase II study was conducted to evaluate the efficacy of combination therapy with TACE and orantinib. The results showed improved progression-free survival (PFS), the primary endpoint of the study, suggesting the additive effect of the combination of orantinib and TACE [14]. Accordingly, a phase III (ORIENTAL) study was conducted in Japan, Korea, and Taiwan [15].

\section{Methods}

Between December 2010 and November 2013, 889 patients with unresectable HCC were registered at 75 institutes located in the 3 countries and subsequently allocated to receive orantinib or placebo. The primary endpoint was OS, and the secondary endpoints were time to TACE failure (TTTF), time to treatment failure, and safety.

\section{Results}

Of 889 patients initially registered, 1 withdrew and the remaining patients were allocated to receive orantinib $(n=444)$ or placebo $(n=444)$. Follow-up was initially planned to continue until November 2016; however, the study was terminated after an interim analysis based on

TACE in Combination with a Molecular

Targeted Agent the recommendation of the Data Monitoring Committee, which provides independent assessment of efficacy and safety, because data analysis suggested that the primary endpoint of the study (OS) would not be achieved. The median OS was 31.1 and 32.3 months in the orantinib and placebo groups, respectively (HR 1.090 [95\% CI 0.8781.352]; $p=0.435$ ) (Table 1). The median OS in patients with BCLC stage B HCC was 31.1 and 26.6 months in the orantinib and placebo groups, respectively (HR 0.837 [95\% CI 0.624-1.124]; $p=0.236$ ). The median TTTF, a secondary endpoint, was 23.9 and 19.8 months in the orantinib and placebo groups, respectively (HR 0.887 [95\% CI 0.725-1.086]; $p=0.245$ ). In addition, the median TTTF in patients with a low level of VEGF-C was 25.5 and 18.4 months in the orantinib and placebo groups, respectively (HR 0.695; $p=0.0196$ ).

\section{Possible Reasons for Failure}

OS, the primary endpoint of this study, did not differ significantly between the groups, whereas TTTF was significantly extended in patients with low levels of VEGF-C in the orantinib group. Additionally, orantinib tended to prolong OS in patients with BCLC stage B HCC, which is a recommended indication for TACE. The study was terminated early because of the interim analysis results that orantinib had no survival benefit in combination with TACE. Because patients with unresectable HCC were targeted in this study, patients with BCLC stage B HCC, the conventional indication for TACE, accounted for only $50 \%$ of all patients. The remaining subjects were BCLC stage A or C. Intergroup comparison after excluding patients with BCLC stage B HCC revealed that OS was poor in the orantinib group, which might have worsened the OS of the entire orantinib group.

Comparison of OS between patients with and without orantinib dose reduction showed that OS was significantly prolonged in patients with dose reduction, whereas OS was shorter in patients without dose reduction than in the placebo group. Comparison by country showed that the proportion of patients with dose reduction was approximately $50 \%$ in Japan, whereas only $30 \%$ of patients had dose reduction in Korea and Taiwan. Side effects were the primary reason for dose reduction in approximately $90 \%$ of patients, suggesting the importance of controlling drug adverse effects. OS tended to be prolonged in the orantinib group compared to the placebo group in Japan, whereas the opposite tendency was observed in Korea and Taiwan. This suggested that OS was longer in Japan because of the proper dose modification and adequate management of side effects.

Oncology 2017;93(suppl 1):127-134 131 


\section{The TACE-2 Study}

\section{Background}

Twenty institutes in England participated in this study, which aimed to clarify whether combination therapy with DEB-TACE and sorafenib suppresses tumor progression and extends OS [16].

\section{Methods}

Patients were allocated to receive sorafenib or placebo, and DEB-TACE was performed within 2-5 weeks after administration of sorafenib or placebo. Subsequently, DEB-TACE was repeated when an additional TACE session was necessary based on imaging findings. The primary endpoint was PFS, and the secondary endpoints were OS and TTP.

\section{Results}

A total of 313 patients were registered for the study and randomized to the sorafenib $(n=157)$ or placebo $(n=156)$ arms. The median PFS per RESIST version 1.1, the primary endpoint of the study, was 7.9 months $(95 \%$ CI 7.4-9.1) and 7.8 months (95\% CI 7.0-10.7) in the sorafenib and placebo arms, respectively (HR 0.99 [95\% CI 0.77-1.27]; $p=0.94)$. The median OS, the secondary endpoint, was 21.1 months (95\% CI 14.7-29.3) and 19.7 months (95\% CI 16.7-23.2) in the sorafenib and placebo groups, respectively (HR 0.91 [95\% CI 0.67-1.24]; $p=$ $0.57)$. The authors concluded that these results, taken together with those from the SPACE trial, provide definitive evidence that DEB-TACE combined with sorafenib therapy does not improve outcome compared to DEBTACE alone.

\section{Possible Reasons for Failure}

In this trial, no significant difference in PFS or OS was observed, showing no beneficial effect of sorafenib. DEBTACE was repeated at the discretion of the physician, even before confirming the progression. Because progression was defined based on RECIST 1.1, sorafenib treatment was terminated at the point of appearance of new intrahepatic lesions. Therefore, it is possible that DEB-TACE was repeated before sorafenib could produce the anticipated benefit, rendering the study results insignificant. The appearance of new intrahepatic lesions does not always indicate that TACE or sorafenib is not effective and should be switched to another treatment option, because intrahepatic metastatic recurrence through the portal vein frequently occurs in HCC. This is a biological characteristic of HCC even after resection, ablation, or superselective TACE in cases in which HCC nodules are large $(>2 \mathrm{~cm})$ [17]. Therefore, the definition of progression in patients undergoing TACE based on RECIST 1.1 or mRECIST is not adequate.

Even in the absence of significant differences between patients treated with TACE alone and those treated with TACE combined with a molecular targeted agent, studies showed the antitumor effect of molecular targeted therapy in an adjuvant setting. However, the TACE- 2 study did not show intergroup differences or an antitumor effect of sorafenib in the adjuvant setting. This was similar to the STORM study, which investigated the efficacy of sorafenib as adjuvant to resection or radiofrequency ablation and found no effect of sorafenib on suppressing recurrence as adjuvant therapy for HCC [18]. These findings suggest that concurrent administration of sorafenib has no effect on the suppression of disease progression based on RECIST and that TACE may be combined with more potent agents, such as immunotherapy or a drug causing a significant response in the tumor, such as lenvatinib [19]. Another possible explanation for the negative results is the trial design, especially the definition of progression. As stated earlier, progression based on RESIST 1.1 or mRECIST may not be adequate in trials of TACE combination therapies [12].

\section{Proposal of a New Primary Endpoint in TACE Combination Trials}

In phase III studies evaluating the effects of cancer treatment, the primary endpoint is OS in principle. However, in previous combination studies with TACE, the median OS ranged from 18 months (shortest) to 32 months (longest) (Table 1), suggesting that the duration of the study needs to be extremely long when evaluating OS as a primary endpoint. In clinical studies that are terminated early because of tumor progression or adverse effects, patients often receive various post-trial treatments, such as hepatic artery infusion chemotherapy [20-22], ablation [23-25], or systemic therapy [26-29]. In such cases, post-trial treatment likely affects OS, making it difficult to evaluate treatment outcomes using OS, especially in studies of TACE in patients with intermediate-stage HCC.

Instead of OS, TTP/PFS is used as a primary endpoint in some trials; however, whether TTP/PFS based on RECIST is an appropriate endpoint in clinical trials of TACE combination therapies remains unclear. In general, TTP corresponds to the period between random-
132

Oncology 2017;93(suppl 1):127-134

DOI: $10.1159 / 000481243$
Kudo/Arizumi 
ization or the day of study initiation and disease progression. However, in patients treated with TACE, the procedure is often repeated before the tumor diameter exceeds the pretreatment diameter. Additionally, patients with indications appropriate for TACE often have multiple lesions and are likely to develop new ones, which leads to the question of whether a new lesion should be considered as PD.

In our previous study, we proposed a novel endpoint, "time to TACE progression (TTTP)," as a progressionfree period specific to patients treated with TACE and defined as the time from the initial TACE effect evaluation to progression [30]. TTTP was determined using images taken 1 month after TACE as baseline images and by designating $\mathrm{PD}$ as the time at which the sum of the diameters of the 5 largest tumors is larger than the baseline diameter by $\geq 20 \%$ or the time of appearance of extrahepatic spread or vascular invasion. TTTP is better suited for evaluating the effect of TACE in clinical practice. We also verified that TTTP is correlated with OS, suggesting that TTTP could be a surrogate endpoint for OS and a better primary endpoint than OS for use in clinical studies of TACE in combination with molecular targeted agents.

\section{Conclusion}

Many clinical trials investigated the efficacy of TACE combined with molecular targeted agents; however, none of them demonstrated an OS benefit of the combination strategy or even improved TTP/PFS. Nevertheless, these studies showed the antitumor effect of the molecular targeted agents as adjuvant therapy. It is important to consider the reasons for the negative outcomes of these trials and to carefully plan future clinical trials of combination therapy with TACE.

\section{Disclosure Statement}

The authors have no conflicts of interest to declare.

\section{References}

1 Llovet JM, Ricci S, Mazzaferro V, Hilgard P, Gane E, Blanc JF, de Oliveira AC, et al: Sorafenib in advanced hepatocellular carcinoma. N Engl J Med 2008;359:378-390.

2 Cheng AL, Kang YK, Chen Z, Tsao CJ, Qin S, Kim JS, Luo R, et al: Efficacy and safety of sorafenib in patients in the Asia-Pacific region with advanced hepatocellular carcinoma: a phase III randomised, double-blind, placebo-controlled trial. Lancet Oncol 2009; 10:25-34.

3 Kudo M: Locoregional therapy for hepatocellular carcinoma. Liver Cancer 2015;4:163164

4 Kudo M: Surveillance, diagnosis, treatment, and outcome of liver cancer in Japan. Liver Cancer 2015;4:39-50.

5 Tsurusaki M, Murakami T: Surgical and locoregional therapy of HCC: TACE. Liver Cancer 2015;4:165-175.

6 Arizumi T, Ueshima K, Minami T, Kono M, Chishina H, Takita M, Kitai S, et al: Effectiveness of sorafenib in patients with transcatheter arterial chemoembolization (TACE) refractory and intermediate-stage hepatocellular carcinoma. Liver Cancer 2015;4:253-262.

7 Bruix J, Raoul JL, Sherman M, Mazzaferro V, Bolondi L, Craxi A, Galle PR, et al: Efficacy and safety of sorafenib in patients with advanced hepatocellular carcinoma: subanalyses of a phase III trial. J Hepatol 2012;57:821829

TACE in Combination with a Molecular Targeted Agent
8 Geschwind JF, Gholam PM, Goldenberg A, Mantry P, Martin RC, Piperdi B, Zigmont E, et al: Use of transarterial chemoembolization (TACE) and sorafenib in patients with Unresectable hepatocellular carcinoma: US regional analysis of the GIDEON registry. Liver Cancer 2016;5:37-46.

9 Kudo M, Imanaka K, Chida N, Nakachi K, Tak WY, Takayama T, Yoon JH, et al: Phase III study of sorafenib after transarterial chemoembolisation in Japanese and Korean patients with unresectable hepatocellular carcinoma. Eur J Cancer 2011;47:2117-2127.

10 Kudo M, Han G, Finn RS, Poon RT, Blanc JF, Yan L, Yang J, et al: Brivanib as adjuvant therapy to transarterial chemoembolization in patients with hepatocellular carcinoma: a randomized phase III trial. Hepatology 2014;60: 1697-1707.

11 Lencioni R, Llovet JM: Modified RECIST (mRECIST) assessment for hepatocellular carcinoma. Semin Liver Dis 2010;30:52-60.

12 Lencioni R, Llovet JM, Han G, Tak WY, Yang J, Guglielmi A, Paik SW, et al: Sorafenib or placebo plus TACE with doxorubicin-eluting beads for intermediate stage HCC: the SPACE trial. J Hepatol 2016;64:1090-1098.

13 Kanai F, Yoshida H, Tateishi R, Sato S, Kawabe T, Obi S, Kondo Y, et al: A phase I/II trial of the oral antiangiogenic agent TSU-68 in patients with advanced hepatocellular carcinoma. Cancer Chemother Pharmacol 2011; 67:315-324.
14 Inaba Y, Kanai F, Aramaki T, Yamamoto T, Tanaka T, Yamakado K, Kaneko S, et al: A randomised phase II study of TSU-68 in patients with hepatocellular carcinoma treated by transarterial chemoembolisation. Eur J Cancer 2013;49:2832-2840.

15 Kudo M, Cheng A-L, Park J-W, Park JH, Liang P-C, Hidaka H, Izumi N, Heo J, Lee YJ, Sheen I-S, Chiu C-F, Arioka H, Morita S, Arai Y: Orantinib versus placebo combined with transcatheter arterial chemoembolization in patients with unresectable hepatocellular carcinoma (ORIENTAL): a randomised, doubleblind, placebo-controlled, multicentre, phase 3 study. Lancet Gastroenterol Hepatol 2017, Epub ahead of print.

16 Meyer T, Fox R, Ma YT, Ross PJ, James MW, Sturgess R, Stubbs C, et al: Sorafenib in combination with transarterial chemoembolisation in patients with unresectable hepatocellular carcinoma (TACE 2): a randomised placebo-controlled, double-blind, phase 3 trial. Lancet Gastroenterol Hepatol 2017;2:565575 .

17 Nakashima O, Sugihara S, Kage M, Kojiro M: Pathomorphologic characteristics of small hepatocellular carcinoma: a special reference to small hepatocellular carcinoma with indistinct margins. Hepatology 1995;22:101-105. 
18 Bruix J, Takayama T, Mazzaferro V, Chau GY, Yang J, Kudo M, Cai J, et al: Adjuvant sorafenib for hepatocellular carcinoma after resection or ablation (STORM): a phase 3, randomised, double-blind, placebo-controlled trial. Lancet Oncol 2015;16:13441354.

19 Cheng A, Finn R, Qin S, et al: Phase III trial of lenvatinib (LEN) vs sorafenib (SOR) in firstline treatment of patients (pts) with unresectable hepatocellular carcinoma (uHCC). J Clin Oncol 2017;35(suppl):abstr 4001.

20 Obi S, Sato S, Kawai T: Current status of hepatic arterial infusion chemotherapy. Liver Cancer 2015;4:188-199.

21 Lin CC, Hung CF, Chen WT, Lin SM: Hepatic arterial infusion chemotherapy for advanced hepatocellular carcinoma with portal vein thrombosis: impact of early response to 4 weeks of treatment. Liver Cancer 2015;4: 228-240.
22 Ueshima K, Kudo M, Tanaka M, Kumada T, Chung H, Hagiwara S, Inoue T, et al: Phase I/ II study of sorafenib in combination with hepatic arterial infusion chemotherapy using low-dose cisplatin and 5-fluorouracil. Liver Cancer 2015;4:263-273.

23 Teng W, Liu KW, Lin CC, Jeng WJ, Chen WT, Sheen IS, Lin CY, et al: Insufficient ablative margin determined by early computed tomography may predict the recurrence of hepatocellular carcinoma after radiofrequency ablation. Liver Cancer 2015;4:26-38.

24 Kang TW, Rhim H: Recent advances in tumor ablation for hepatocellular carcinoma. Liver Cancer 2015;4:176-187.

25 Lencioni R, de Baere T, Martin RC, Nutting CW, Narayanan G: Image-guided ablation of malignant liver tumors: recommendations for clinical validation of novel thermal and non-thermal technologies - a Western perspective. Liver Cancer 2015;4:208-214.
26 Kudo M: Molecular targeted therapy for hepatocellular carcinoma: where are we now? Liver Cancer 2015;4:I-VII.

27 Kudo M: Regorafenib as second-line systemic therapy may change the treatment strategy and management paradigm for hepatocellular carcinoma. Liver Cancer 2016;5:235-244.

28 Zhang B, Finn RS: Personalized clinical trials in hepatocellular carcinoma based on biomarker selection. Liver Cancer 2016;5:221232.

29 Kudo M: Immune checkpoint blockade in hepatocellular carcinoma. Liver Cancer 2015;4: 201-207.

30 Arizumi T, Ueshima K, Iwanishi M, Minami T, Chishina H, Kono M, Takita M, et al: The overall survival of patients with hepatocellular carcinoma correlates with the newly defined time to progression after transarterial chemoembolization. Liver Cancer 2017;6: 227-235. 